\title{
The Association Between Training Load and Injury Risk in Elite Youth Soccer Players: a Systematic Review and Best Evidence Synthesis
}

Sven Verstappen ${ }^{1,2,3}$, Rogier M. van Rijn ${ }^{1,2}$, Rick Cost $^{4}$ and Janine H. Stubbe $1,2,5,6^{*}$

\begin{abstract}
Background: Injury risk in elite youth soccer players is high. Implementing an optimal training load is of utmost importance to reduce the risk of injuries.

Objective: To conduct a systematic review and best evidence synthesis to explore the effects of internal and external training load on injury risk in elite youth soccer players.

Methods: MEDLINE, Embase, Web of Science, CENTRAL, and CINAHL were searched up until 17 January 2020. Each article had to meet all of the following criteria: (1) the study population consisted of male elite youth soccer players aged between 12 and 21 years; (2) a longitudinal, prospective study design was used; (3) soccer-related injuries were registered (i.e., self-reported or by medical staff); (4) external and/or internal load parameters were described; and (5) the article was published in an English peer-reviewed scientific journal. The quality of the included articles was assessed using the Newcastle-Ottawa Quality Assessment Scale (NOS). A best evidence synthesis was performed to rank the level of evidence.

Results: Five studies (2 high quality, 3 low quality) were included. Best evidence synthesis highlighted that there was moderate evidence for (1) no association between 2-, 3-, and 4-week cumulative loads for total distance covered; (2) no association between 1-week workloads (sRPE $\times$ duration); and (3) no association between A:C workload ratios (4 weeks) and injury risk. For all other comparisons, only insufficient or conflicting evidence was found.
\end{abstract}

Conclusion: There is a paucity of evidence for an association between internal and external training load parameters and injury risk in elite youth soccer players.

Keywords: Workload, Injury, Football, Adolescent, Youth, Risk factor

\footnotetext{
*Correspondence: jhstubbe@codarts.nl

${ }^{1}$ Codarts, University of the Arts, Kruisplein 26, 3012 CC Rotterdam, The

Netherlands

${ }^{2}$ PErforming artist and Athlete Research Lab (PEARL), Rotterdam, The

Netherlands

Full list of author information is available at the end of the article
}

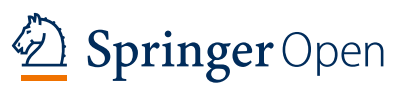

(c) The Author(s). 2021 Open Access This article is licensed under a Creative Commons Attribution 4.0 International License, which permits use, sharing, adaptation, distribution and reproduction in any medium or format, as long as you give appropriate credit to the original author(s) and the source, provide a link to the Creative Commons licence, and indicate if changes were made. The images or other third party material in this article are included in the article's Creative Commons licence, unless indicated otherwise in a credit line to the material. If material is not included in the article's Creative Commons licence and your intended use is not permitted by statutory regulation or exceeds the permitted use, you will need to obtain permission directly from the copyright holder. To view a copy of this licence, visit http://creativecommons.org/licenses/by/4.0/. 


\section{Key Points}

- There is a lack of evidence for an association between internal and external training load parameters and injury risk in elite youth soccer players.

- Future high-quality longitudinal research is required to further assess the role of internal and external training load on the occurrence of injuries in elite youth soccer players, where a multifactorial approach must also be applied.

\section{Introduction}

Soccer has evolved and games are played faster and more aggressively than in the past, requiring elevated fitness levels [1]. To meet these physical demands, soccer players have to be exposed to systematic and appropriate training regimes taking into account the balance between training load (TL; the combination of training volume, intensity, and frequency) and recovery [2]. TLs that are too low are insufficient to induce a functional, adaptive response. However, training too hard increases the risk of health problems, such as overtraining and injuries [3]. The high injury incidence in professional soccer players, ranging from 2.48 injuries [4] to 9.4 injuries [5] per $1000 \mathrm{~h}$ of exposure, suggests that finding the right balance between training, matches, and recovery is one of the biggest challenges soccer coaches and their staff face.

Adolescence, the period of life between childhood and adulthood, is a critical time in a soccer player's career [6], and during this period, injuries can have a detrimental effect on future performance $[7,8]$ and career opportunities [9-12]. Unfortunately, injury risk in elite youth soccer players is even higher compared to their professional counterparts, ranging from 2.0 [13] to 19.4 injuries [14] per $1000 \mathrm{~h}$ of exposure, with match injury incidence as high as 48.7 injuries [14] per $1000 \mathrm{~h}$ of match exposure.

Implementing an optimal training load in elite youth soccer training programs is of utmost importance to reduce the risk of injuries and attain peak performance. Training load can be categorized into two components. External load refers to the physical work performed during training or matches, such as distance covered and accelerations [15], whereas internal load comprises the psychophysiological response to the external load [15]. Several studies in elite male soccer players have investigated the association between load and injury risk and concluded that players have an increased injury risk if the training load exceeds what their bodies can tolerate [16-20]. This association has received less attention in elite youth soccer players. While training may provoke positive training adaptations in youth athletes, it is also likely that they will respond differently from their adult counterparts to a particular training load, resulting in different fatigue, stress, injury, or illness responses [6]. Gabbett et al. determined the existing knowledge on the relationship between workload and injuries in adolescent male football players (i.e., American football, Australian rules football, soccer, rugby league, rugby union) and concluded that the balance between what is required to maintain or improve skill versus physiological performance and the association with injury risk is not well understood [6]. Therefore, the aim of our study was to conduct a systematic review exploring the effects of external and internal training load on injury risk in elite youth soccer players. This enables coaches and staff to better understand the impact of training load on injury risk in this specific target group.

\section{Methods}

\section{Search Strategy}

The systematic review was conducted according to the Preferred Reporting Items for Systematic Reviews and Meta-Analyses (PRISMA) guidelines [21]. Systematic literature searches were conducted in MEDLINE, Embase, Web of Science, the Cochrane Central Register of Controlled Trials (CENTRAL), and CINAHL (from the inception of databases up to 17 January 2020). The full search strategy is presented in Supplementary File 1.

Based on the title and abstract, two reviewers (SV, RMvR) selected the articles for full-text appraisal. From this pool, the two reviewers (SV, RMvR) independently selected articles for final inclusion. Each article had to meet all of the following criteria: (1) the study population consisted of male elite youth soccer players aged between 12 and 21 years, (2) a longitudinal prospective study design was used, (3) soccer-related injuries were registered (i.e., self-reported or by medical staff), (4) external and/or internal load parameters were described, and (5) the article was published in an English peerreviewed scientific journal. Elite youth soccer players were defined as players who are part of an academy of an elite soccer club playing in the highest competition of their country. EndNote X8 software (Clarivate Analytics, Philadelphia, PA, USA) was used to perform the selection process. Any disagreement over inclusion was resolved via discussion between the reviewers. In the case of disagreement, a third reviewer (JHS) was consulted. The references of all included studies were checked for other relevant articles.

\section{Data Extraction}

The following relevant data from each study were extracted: study details (author, year of publication, country, duration of follow-up), study population (sample size, age), injury definition, workload (external and internal load parameters), and measure of association (i.e., relative risk $[R R]$ or odds ratio $[\mathrm{OR}])$. Where possible, 
these associations were directly extracted from the original article. For articles in which this information was not presented, associations calculated using raw data were provided. Load parameters were classified as external or internal based on the International Olympic Committee consensus statement on load in sport and risk of injury [22]. The first reviewer (SV) extracted data from the included studies. In case of uncertainty, a second reviewer (RMvR) was consulted.

\section{Quality Assessment}

The methodological quality of each study was assessed using an adapted version of the NewcastleOttawa Quality Assessment Scale (NOS) for cohort studies [23]. The original NOS consists of eight items and judges the quality of case-control and cohort studies. For cohort studies, the eight items can be grouped into three perspectives: selection of cohorts (4 items), comparability of cohorts ( 1 item), and assessment of outcome (3 items). A star-based rating system is used to indicate the quality of a study. A maximum of one star can be given for each item within the "Selection" and "Outcome" categories, and a maximum of two stars for the "Comparability" category, resulting in a maximum score of nine stars for high-quality studies. The original NOS was modified for the purpose of our review. In our modified version, two items of the original eight were deleted. Items 2 (selection of a non-exposed cohort) and 5 (assesses the comparability of cohorts) were deleted as our review focuses solely on male elite youth soccer players. In addition, we included a new item to the original scale, regarding injury definition, which was adopted from a modified version of the NOS used in a systematic review and meta-analysis of epidemiological data on injuries in professional male soccer [24]. Table 1 describes the seven criteria of our adapted version of the Newcastle-Ottawa Scale (NOS) for cohort studies. A study could be awarded a maximum of one star for each item if appropriate methods had been clearly reported, resulting in a maximum score of seven stars. The higher the number of stars given to a study, the lower the risk of bias. NOS scores were divided into high quality/low risk of bias (5-7 stars) and low quality/high risk of bias (0-4 stars).

Each included study was appraised by two authors (SV and RMvR) independently, and all discrepancies in scoring were resolved by arbitration between the two reviewers. In cases where discrepancies could not be resolved, a third reviewer (JHS) assessed the item in question. The reviewed studies were not blinded for reasons of practicality.

A best evidence synthesis was conducted to rate the strength of the evidence. The following ranking of evidence was used [25]:

1) Strong evidence: consistent findings in multiple ( $\geq$ 2) high-quality studies;

2) Moderate evidence: consistent findings in one highquality study and at least one low-quality study, or consistent findings in multiple low-quality studies;

3) Insufficient evidence: only one study available; and

4) Conflicting evidence: inconsistent findings in multiple $(\geq 2)$ studies.

Results of the studies reporting on a particular relationship were considered consistent when, for at least

Table 1 Description of the 7 criteria designed to assess the risk of bias of external validity quality in the studies

\begin{tabular}{|c|c|}
\hline Criterion & Description of criteria \\
\hline 1. Definition of soccer-related injury & $\begin{array}{l}\text { Studies that aimed to investigate soccer-related injuries should present a definition of an in- } \\
\text { jury informing what was considered as an injury in the study. } \\
\text { Studies that present a definition of time-loss injury received a star for this criterion. }\end{array}$ \\
\hline 2. Representativeness of the exposed cohort & $\begin{array}{l}\text { (a) Truly representative of the average soccer players in the community }{ }^{a} \text {; (b) somewhat } \\
\text { representative of the average soccer players in the community }{ }^{a} ;(c) \text { selected group of users; } \\
\text { (d) no description of the derivation of the cohort. }\end{array}$ \\
\hline 3. Ascertainment of exposure & (a) Secure record ; ; b) structured interview ${ }^{\mathrm{a}}$; (c) written self-report; (d) no description. \\
\hline $\begin{array}{l}\text { 4. Demonstration that outcome of interest was not } \\
\text { present at the start of the study }\end{array}$ & $\begin{array}{l}\text { (a) Yes }{ }^{a} \text {; (b) no } \\
\text { Studies that described that all soccer players included were injury-free at baseline received a } \\
\text { star for this criterion. }\end{array}$ \\
\hline 5. Assessment of outcome & (a) Independent blind assessment ${ }^{a}$; (b) record linkage ; (c) self-report; (d) no description. \\
\hline $\begin{array}{l}\text { 6. Was follow-up long enough for outcomes to } \\
\text { occur? }\end{array}$ & $\begin{array}{l}\text { (a) Yes }{ }^{\mathrm{a}} \text {; (b) no } \\
\text { Studies that carried out a follow-up period of at least } 12 \text { weeks received a star for this } \\
\text { criterion. }\end{array}$ \\
\hline 7. Adequacy of follow-up of cohorts & $\begin{array}{l}\text { (a) Complete follow-up of all subjects accounted for }{ }^{a} \text {; (b) subjects lost to follow-up unlikely to } \\
\text { introduce bias (up to } 20 \% \text { loss) or description provided of those lost }{ }^{a} \text {; (c) follow-up rate }<80 \% \\
\text { and no description of those lost; (d) no statement. }\end{array}$ \\
\hline
\end{tabular}


$75 \%$ of the study, results were in the same direction, as defined by $p<0.05$.

\section{Results \\ Search Results}

An initial 12,501 articles were retrieved from database searches $($ Embase $=3393$; MEDLINE Ovid $=3643 ; \mathrm{Web}$ of Science $=3014$; Cochrane CENTRAL $=303$; CINAHL = 2148). After removal of 5944 duplicate records, a further 6557 irrelevant articles were removed based on title and abstract, resulting in 44 articles for full-text appraisal. Finally, five articles were included in the review (see Fig. 1).

\section{Description of the Included Studies}

The five included studies accounted for a total participant pool of 270 elite male youth soccer players [26-30]. Follow-up periods ranged from one [30] to five [29] consecutive seasons. The sample size ranged from 22 to 122 soccer players. Two studies included external load measurements $[26,27]$, and three studies examined both external and internal load parameters [28-30]. The following load parameters were taken into account in the included studies: (1) internal load: psychosocial stress and recovery; (2) external load: total distance covered, high speed running, acceleration, duration, monotony, acute to chronic (AC) ratio (based on GPS and accelerometer-derived variables); and (3) combination of internal and external load parameters: workload, strain, and $\mathrm{AC}$ ratio (based on sRPE and duration). In four (out of five) studies, an injury definition was described. Definitions provided were (1) any physical complaint sustained by a player that results from a soccer match or training, resulting in time loss (unable to take full part in future soccer activities) or medical attention ( $>1$ day but still able to take full part in future soccer activities) [28], and (2) injury that occurred during a scheduled training session or match that caused absence from the next training session or match $[27,29,30]$. In three studies, injuries were registered by the medical staff [26-28], in one study by self-report [30], and in one study no description was provided [29]. A detailed description of the study characteristics is presented in Table 2 .

\section{Risk of Bias Assessment and Best Evidence Synthesis}

Of the five included studies, two were of high quality (low risk of bias) and three were of low quality (high risk of bias) (Table 3). Reviewers (SV, RMvR) retained agreement on all scoring and bias assessment results. For full details of the best evidence synthesis, see Tables 4, 5, and 6 .

\section{Evaluation of Internal Load Parameters Psychosocial Stress and Recovery}

Insufficient evidence exists for an association between psychosocial stress and recovery, and traumatic and overuse injury risk [28]. This high-quality study used the Recovery Stress Questionnaire for Athletes (RESTQSport77) and concluded that the subscale fitness/injury was associated with the occurrence of traumatic and

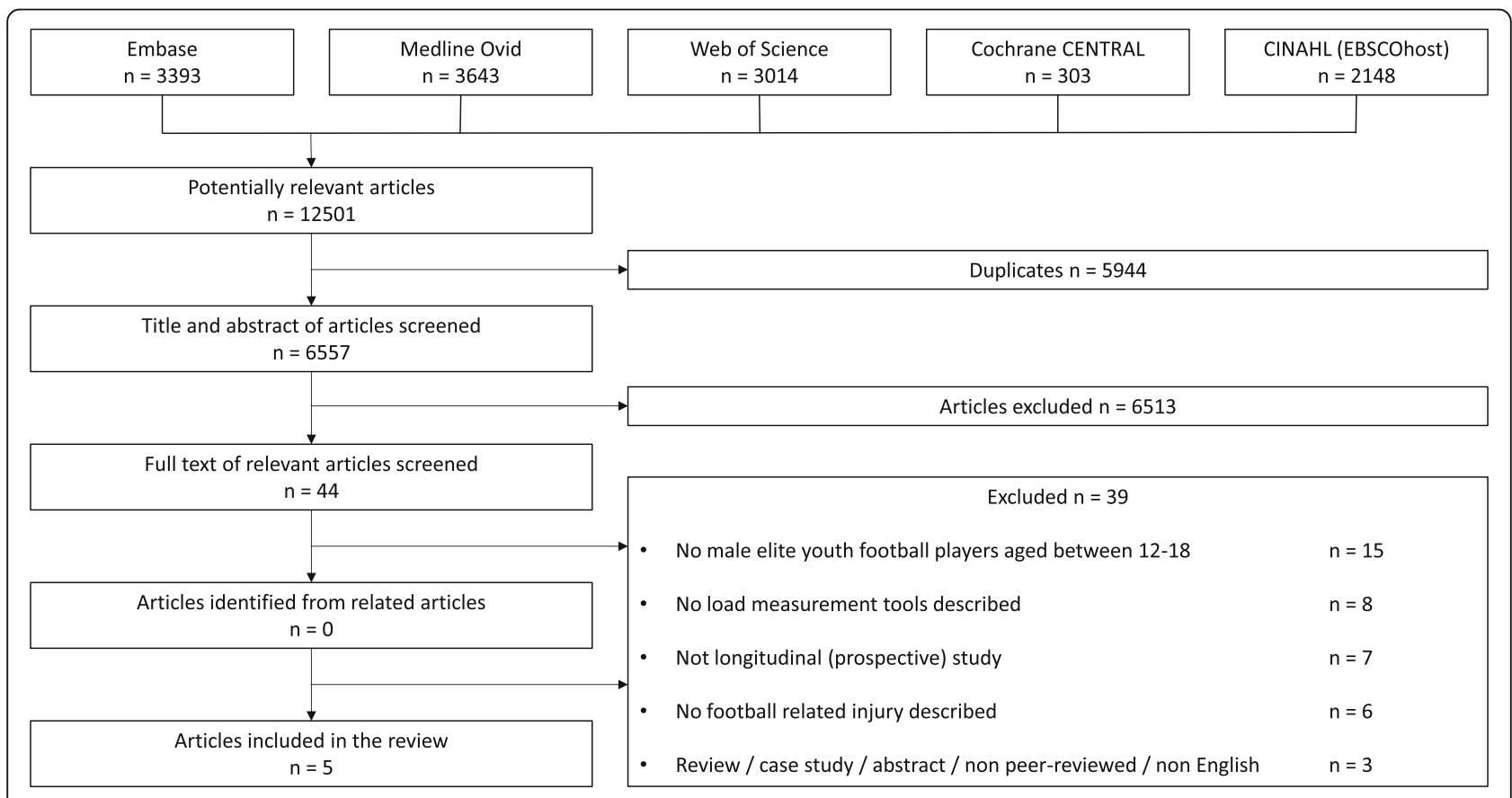

Fig. 1 Flowchart of the selection process for inclusion of articles in the systematic review 
Table 2 Characteristics of included studies

\begin{tabular}{|c|c|c|c|c|c|}
\hline \multirow[t]{2}{*}{ Study } & \multirow{2}{*}{$\begin{array}{l}\text { Follow- } \\
\text { up }\end{array}$} & \multirow[t]{2}{*}{ Population } & \multirow[t]{2}{*}{ Injury definition } & \multicolumn{2}{|l|}{ Workload } \\
\hline & & & & Internal & External \\
\hline $\begin{array}{l}\text { Bacon and } \\
\text { Mauger } \\
{[26]} \\
2017 \\
\text { UK }\end{array}$ & $\begin{array}{l}2 \\
\text { seasons }\end{array}$ & $\begin{array}{l}N=41 \\
\text { Mean age } \\
17.8 \pm 1.1 \\
\text { yrs }\end{array}$ & $\begin{array}{l}\text { Overuse injuries-no definition } \\
\text { described }\end{array}$ & n.a. & $\begin{array}{l}\text { Total distance }(\mathrm{m}) \text {; weekly and whole } \\
\text { season training/match averages } \\
\text { High speed running }(\mathrm{m}) \text {; weekly and } \\
\text { whole season training/match averages }\end{array}$ \\
\hline $\begin{array}{l}\text { Bowen } \\
\text { et al. [27] } \\
2017 \\
\text { UK }\end{array}$ & $\begin{array}{l}2 \\
\text { seasons }\end{array}$ & $\begin{array}{l}N=32 \\
\text { Mean age } \\
17.3 \pm 0.9 \\
\text { yrs }\end{array}$ & $\begin{array}{l}\text { A non-contact/contact injury was de- } \\
\text { fined as one that caused any absence } \\
\text { from future football participation }\end{array}$ & n.a. & $\begin{array}{l}\text { Total distance }(\mathrm{m}) \text {; this includes } \\
\text { walking, jogging, fast running, and } \\
\text { sprinting } \\
\text { High speed distance }(\mathrm{m}) \text {; total } \\
\text { distance }>20 \mathrm{~km} / \mathrm{h} \\
\text { Total load (au); acceleration along } x, y \text {, } \\
\text { and } z \text { axes } \\
\text { Accelerations }(n) \text {; a change in GPS } \\
\text { speed data for at least half a second } \\
\text { with maximum acceleration in the } \\
\text { period of at least } 0.5 \mathrm{~m} / \mathrm{s} / \mathrm{s}\end{array}$ \\
\hline $\begin{array}{l}\text { Brink et al. } \\
\text { [28] } \\
2010 \\
\text { Netherlands }\end{array}$ & $\begin{array}{l}2 \\
\text { seasons }\end{array}$ & $\begin{array}{l}N=53 \\
\text { Mean age } \\
15-18 \text { yrs }\end{array}$ & $\begin{array}{l}\text { Any physical complaint sustained by a } \\
\text { player that results from a soccer match } \\
\text { or training, resulting in time loss (unable } \\
\text { to take full part in future soccer } \\
\text { activities) or medical attention (> } 1 \text { day } \\
\text { but still able to take full part in future } \\
\text { soccer activities) } \\
\text { Traumatic injuries resulted from a } \\
\text { specific, identifiable event. Overuse }\end{array}$ & $\begin{array}{l}\text { Rate of perceived exertion; } \\
\text { global intensity of each } \\
\text { training session rated on } \\
\text { Borg } 15 \text {-point scale } \\
\text { Psychosocial stress and } \\
\text { recovery; Recovery Stress } \\
\text { Questionnaire for athletes } \\
\text { (RESTQ-Sport) }\end{array}$ & $\begin{array}{l}\text { Physical stress (min); duration of } \\
\text { training sessions and matches } \\
\text { Training load; product of the session- } \\
\text { RPE and the physical stress } \\
\text { Monotony; daily mean training load } \\
\text { divided by the SD of the daily mean } \\
\text { training load over a 1-week period } \\
\text { Strain; product of weekly training load } \\
\text { and monotony }\end{array}$ \\
\hline
\end{tabular}

\begin{tabular}{|c|c|c|c|}
\hline $\begin{array}{l}\text { Delecroix } \\
\text { et al. [29] } \\
2019 \\
\text { France }\end{array}$ & $\begin{array}{l}4(\cup 19) \\
\text { and } 5 \\
(\cup 21) \\
\text { seasons }\end{array}$ & $\begin{array}{l}\text { U19: } n= \\
\text { 52; mean } \\
\text { age } 16.8 \pm \\
0.9 \text { yrs } \\
\text { U21: } n= \\
\text { 70; mean } \\
\text { age } 20.1 \pm \\
0.3 \text { yrs }\end{array}$ & $\begin{array}{l}\text { Any physical complaint sustained by a } \\
\text { player that resulted from a football } \\
\text { match or training, that made the player } \\
\text { unable to participate in a future football } \\
\text { training or match }\end{array}$ \\
\hline $\begin{array}{l}\text { Raya- } \\
\text { González } \\
\text { et al. [30] } \\
2019\end{array}$ & $\begin{array}{l}1 \\
\text { season }\end{array}$ & $\begin{array}{l}N=22 \\
\text { Mean age } \\
18.6 \pm 0.6 \\
\text { yrs }\end{array}$ & $\begin{array}{l}\text { A non-contact injury that occurred dur- } \\
\text { ing a scheduled training session or } \\
\text { match that caused absence from the } \\
\text { next training session or match }\end{array}$ \\
\hline
\end{tabular}

Rate of perceived exertion; global intensity of each -point scale Questionnaire for athletes specific, identifiable event. Overuse injuries resulted from repeated microtrauma without a single microtrauma with
identifiable event

Rate of perceived exertion; global intensity of each training session and match rated on a 0 -10-point scale

Rate of perceived exertion; global intensity of each training session and match rated on a 0 -10-point scale
Workload (au); product of the sessionRPE and duration of training session and matches

A:C ratio (au); 1-week workload divided by the average workload of the last 28/21/14 days

Week-to-week load changes; 1-week load divided by accumulated load of previous 7 days

Workload (au); the sum of load (RPE* duration) for all training sessions and matches for each week

$A: C$ ratio; sum of the current week's load (acute load) divided by the average weekly training load over the previous four weeks (chronic load)

yrs years, n.a. not applicable, $m$ meters, $k m / h$ kilometers per hour, GPS Global Positioning System, au arbitrary unit, $n$ number, min minutes, RESTQ Recovery Stress questionnaire, $S D$ standard deviation, RPE Rate of Perceived Exertion, A:C ratio acute to chronic workload ratio

Table 3 Quality of included studies as assessed on the Newcastle-Ottawa Scale (NOS)

\begin{tabular}{|c|c|c|c|c|c|c|c|c|c|}
\hline \multirow[t]{3}{*}{ Study } & \multicolumn{8}{|c|}{ NOS score } & \multirow{3}{*}{$\begin{array}{l}\text { Risk } \\
\text { of } \\
\text { bias }\end{array}$} \\
\hline & \multicolumn{4}{|c|}{ Selection } & \multicolumn{3}{|c|}{ Outcome } & \multirow[t]{2}{*}{ Total } & \\
\hline & 1 & 2 & 3 & 4 & 5 & 6 & 7 & & \\
\hline Bacon and Mauger [26] & 0 & 1 & 1 & 0 & 1 & 1 & 0 & 4 & High \\
\hline Bowen et al. [27] & 1 & 1 & 1 & 0 & 1 & 1 & 0 & 5 & Low \\
\hline Brink et al. [28] & 1 & 1 & 1 & 0 & 1 & 1 & 0 & 5 & Low \\
\hline Delecroix et al. [29] & 1 & 1 & 1 & 0 & 0 & 1 & 0 & 4 & High \\
\hline Raya-Gonzales et al. [30] & 1 & 1 & 1 & 0 & 0 & 1 & 0 & 4 & High \\
\hline
\end{tabular}

overuse injuries. Insufficient evidence also exists for a lack of an association between the sum score of the RESTQ-Sport, the subscale stress, and the subscale recovery and traumatic and overuse injury risks.

\section{Evaluation of External Load Parameters Total Distance Covered}

One high-quality study provided insufficient evidence for a lack of an association between total distance (TD) covered and injury risk (contact/traumatic) [27]. Two studies, one high quality and one low quality, investigated the association between TD covered and the occurrence of non-contact/overuse injuries [26, 27]. Both 
Table 4 Results of the best evidence synthesis concerning contact and traumatic injuries

\begin{tabular}{|c|c|c|c|c|c|}
\hline \multirow[t]{2}{*}{ Risk factor } & \multirow[t]{2}{*}{ Study } & \multirow[t]{2}{*}{ Specification of independent variable } & \multirow{2}{*}{$\begin{array}{l}\text { Outcome } \\
\text { OR/RR } \\
(95 \% \mathrm{Cl})\end{array}$} & \multicolumn{2}{|c|}{ Best evidence synthesis } \\
\hline & & & & $\begin{array}{l}\text { Presence of } \\
\text { association }\end{array}$ & $\begin{array}{l}\text { Level of } \\
\text { evidence }\end{array}$ \\
\hline \multicolumn{6}{|l|}{ Internal load parameters } \\
\hline $\begin{array}{l}\text { Psychosocial stress and } \\
\text { recovery }\end{array}$ & $\begin{array}{l}\text { Brink } \\
\text { et al. [28] }\end{array}$ & $\begin{array}{l}\text { General stress } \\
\text { Emotional stress } \\
\text { Social stress } \\
\text { Conflicts/pressure } \\
\text { Fatigue } \\
\text { Lack of energy } \\
\text { Physical complaints } \\
\text { Success } \\
\text { Social recovery } \\
\text { Physical recovery } \\
\text { General well-being } \\
\text { Sleep quality } \\
\text { Disturbed breaks } \\
\text { Emotional exhaustion } \\
\text { Fitness/injury } \\
\text { Being in shape } \\
\text { Personal accomplishment } \\
\text { Self-efficacy } \\
\text { Self-regulation }\end{array}$ & $\begin{array}{l}0.97(0.74-1.27) \\
1.04(0.76-1.44) \\
0.96(0.70-1.30) \\
0.95(0.74-1.22) \\
0.93(0.72-1.20) \\
0.94(0.70-1.26) \\
0.99(0.74-1.33) \\
1.04(0.81-1.35) \\
0.99(0.78-1.26) \\
0.88(0.69-1.13) \\
1.10(0.85-1.42) \\
0.99(0.77-1.27) \\
0.94(0.72-1.22) \\
0.85(0.67-1.09) \\
1.29(1.01-1.66) \\
0.91(0.73-1.13) \\
1.06(0.84-1.35) \\
1.00(0.80-1.25) \\
0.90(0.75-1.08)\end{array}$ & $\begin{array}{l}\text { No } \\
\text { No } \\
\text { No } \\
\text { No } \\
\text { No } \\
\text { No } \\
\text { No } \\
\text { No } \\
\text { No } \\
\text { No } \\
\text { No } \\
\text { No } \\
\text { No } \\
\text { No } \\
\text { Yes } \\
\text { No } \\
\text { No } \\
\text { No } \\
\text { No }\end{array}$ & Insufficient \\
\hline
\end{tabular}

External load parameters

Total distance

Bowen et al. [27]

High speed running

Bowen et al. [27]

HSD—low

HSD — moderately low

HSD — moderately high

HSD—high

HSD—very high

Acceleration Bowen et al. [27]

Accumulated 1/2/3/4-week load

ACC-low

ACC - moderately low

ACC - moderately high

ACC - high

ACC - very high

Duration

Brink et al. [28]

Total load

Bowen et al. [27]

Duration (min) of training sessions and matches

Accumulated 1/2/3/4-week load

$\mathrm{TL}$-low

$\mathrm{TL}$ - moderately low

TL-moderately high

$T L$ - high

TL-very high

Acute to chronic Bowen workload ratio (ACWR) et al. [27]
Insufficient

$$
\begin{array}{ll}
\text { 0.83/0.76/0.84/1.04 } & \text { No/no/no/no } \\
0.68 / 0.65 / 0.87 / 0.92 & \text { No/no/no/no } \\
\text { 0.98/1.62/0.84/0.88 } & \text { No/no/no/no } \\
1.79 / 1.00 / 1.35 / 1.49 & \text { No/no/no/no } \\
-/-/-/- & \text { n.a }
\end{array}
$$

Insufficient

0.79/0.91/0.83/1.14 No/no/no/no 0.41/0.67/0.78/0.97 No/no/no/no 1.74/1.70/1.24/0.68 No/no/no/no 1.08/0.86/1.13/1.74 No/no/no/no 1.97/-/1.62/- No/n.a./no/n.a.

Insufficient

0.72/0.75/0.84/1.02 No/no/no/no 0.65/0.67/1.24/0.91 No/no/no/no $1.39 / 1.51 / 0.76 / 0.92 \mathrm{No} / \mathrm{no} / \mathrm{no} / \mathrm{no}$ $1.27 / 1.06 / 1.49 / 1.35 \mathrm{No} / \mathrm{no} / \mathrm{no} / \mathrm{no}$ -/-/1.02/- n.a./n.a./no/ n.a.

$1.14(1.06-1.23) \quad$ Yes

Insufficient Insufficient

0.77/0.81/0.87/1.06 No/no/no/no 0.63/0.70/1.34/0.98 No/no/no/no $1.12 / 1.76 / 0.77 / 0.89 \mathrm{No} / \mathrm{no} / \mathrm{no} / \mathrm{no}$ 1.42/0.33/0.95/1.43 No/no/no/no 4.84/3.04/2.68/- Yes/no/no/no
$0.37 / 0.26 / 0.62$

$1.72 / 2.12 / 1.47$

$0.44 / 0.44 / 0.91$

$1.22 / 2.80 / 0.9$

4.98/-/3.79
No/no/no No/no/no No/no/no $\mathrm{No} / \mathrm{no} / \mathrm{no}$ Yes/n.a./no 
Table 4 Results of the best evidence synthesis concerning contact and traumatic injuries (Continued)

\begin{tabular}{|c|c|c|c|c|c|}
\hline \multirow[t]{2}{*}{ Risk factor } & \multirow[t]{2}{*}{ Study } & \multirow[t]{2}{*}{ Specification of independent variable } & \multirow{2}{*}{$\begin{array}{l}\text { Outcome } \\
\text { OR/RR } \\
(95 \% \mathrm{CI})\end{array}$} & \multicolumn{2}{|c|}{ Best evidence synthesis } \\
\hline & & & & $\begin{array}{l}\text { Presence of } \\
\text { association }\end{array}$ & $\begin{array}{l}\text { Level of } \\
\text { evidence }\end{array}$ \\
\hline & & 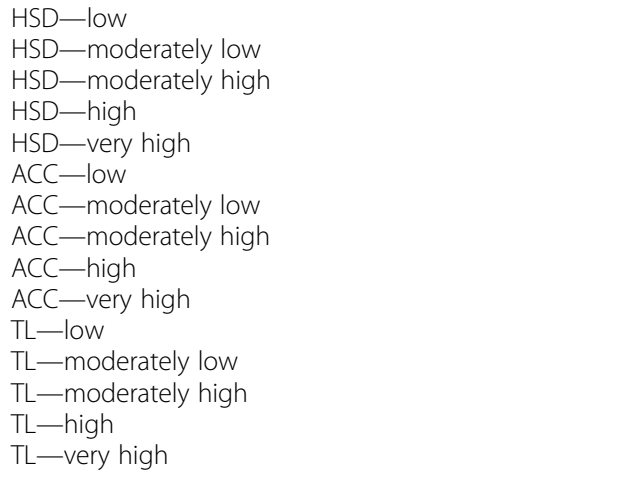 & $\begin{array}{l}0.32 / 0.24 / 1.91 \\
1.45 / 0.82 / 1.52 \\
1.32 / 2.55 / 0.69 \\
0.49 / 0.85 / 0.54 \\
2.28 /-/ 3.62 \\
0.39 / 0.25 /- \\
1.75 / 1.79 / 1.66 \\
0.79 / 0.59 / 1.03 \\
1.47 / 2.48 / 0.64 \\
4.98 /-/ 5.91 \\
0.38 / 0.21 / 0.60 \\
1.92 / 1.64 / 1.97 \\
0.87 / 0.77 / 0.86 \\
1.20 / 2.280 .32 \\
2.74 /-/ 6.12\end{array}$ & $\begin{array}{l}\text { No/no/no } \\
\text { No/no/no } \\
\text { No/no/no } \\
\text { No/no/no } \\
\text { No/no/no } \\
\text { No/no/n.a. } \\
\text { No/no/no } \\
\text { No/no/no } \\
\text { No/no/no } \\
\text { Yes/n.a./no } \\
\text { No/no/no } \\
\text { yes/no/no } \\
\text { No/no/no } \\
\text { No/no/no } \\
\text { No/n.a./no }\end{array}$ & \\
\hline \multicolumn{6}{|c|}{ Combined internal/external load parameters } \\
\hline Workload & $\begin{array}{l}\text { Brink } \\
\text { et al. [28] }\end{array}$ & Product of the session-RPE and duration & $1.01(1.00-1.02)$ & Yes & Insufficient \\
\hline Monotony & $\begin{array}{l}\text { Brink } \\
\text { et al. [28] }\end{array}$ & $\begin{array}{l}\text { Daily mean training load divided by the SD of the daily } \\
\text { mean training load over a } 1 \text {-week period }\end{array}$ & $2.59(1.22-5.50)$ & Yes & Insufficient \\
\hline Strain & $\begin{array}{l}\text { Brink } \\
\text { et al. [28] }\end{array}$ & Product of weekly training load and monotony & $1.01(1.00-1.01)$ & Yes & Insufficient \\
\hline
\end{tabular}

In bold are statistically significant $(p<0.05)$ associations

$O R$ odds ratio, $R R$ risk ratio, $C I$ confidence interval, $T D$ total distance, HSD high speed distance, $A C C$ acceleration, $T L$ total load, min minutes, $A C W R$ acute to chronic workload ratio, n.a. not applicable, RPE rate of perceived exertion, $S D$ standard deviation

studies found no significant effect of 2-, 3-, and 4-week cumulative loads for TD on injury risk, resulting in moderate evidence for no association between these factors. Being in a higher TD loading group lowered the risk of an overuse injury, although insufficient evidence for this relationship exists [26]. Insufficient evidence also exists for a low, 1-weekly TD covered (0-8811 m) reducing the risk of non-contact/overuse injuries [27]. A low, 1-weekly TD $(0-8811 \mathrm{~m})$ reduced the risk of overall injury in one study only, therefore offering insufficient evidence. A moderately high, 2-weekly TD covered (39, 806-58,405 m) and a high 4-weekly TD covered (112, $244-143,917 \mathrm{~m}$ ) increased the risk of overall injury, but this was also only found in one study resulting in insufficient evidence [27]. For all other comparisons, insufficient evidence was found for a lack of association.

\section{High Speed Running}

Insufficient evidence exists for a lack of a relationship between high speed running distance (HSD), accumulated over 1-4 weeks, and the occurrence of contact/ traumatic injuries [27].

Two studies, one high quality and one low quality, investigated the association between HSD and noncontact/overuse injury risk [26, 27]. Being in a higher HSD loading group lowered the risk of an overuse injury, although this was only found in one study leading to insufficient evidence for this effect [26]. Two-,
3-, and 4-week cumulative loads of HSD were not significantly associated with an increased risk of overuse injury in the study of Bacon et al. [26]. A study by Bowen and colleagues showed that low 2-weekly $(0-755 \mathrm{~m})$ and moderately high 4-weekly HSD (3502$5122 \mathrm{~m}$ ) was significantly related with non-contact/ overuse injury occurrence [27], whereas no association was found for 3-weekly HSD loads [27]. Therefore, there is conflicting evidence for an association between 2- and 4-week accumulated HSD loads and non-contact/overuse injury risk, and moderate evidence for no association between 3-week accumulated HSD loads and injury occurrence. There is insufficient evidence for an association between moderately high 1-weekly HSD loads (856-1448 m) and noncontact/overuse injury risk. There is also insufficient evidence for an association between (1) low 1- and 2weekly HSD loads; (2) moderately high 1-, 2-, and 4weekly HSD loads; and (3) high 3-weekly HSD loads and the occurrence of overall injuries [27].

\section{Acceleration}

One high-quality study investigated the relationship between the number of accelerations (ACC) and injury occurrence [27]. Insufficient evidence was found for no association between ACC, accumulated over 1-4 weeks, and the occurrence of contact/traumatic injuries. Insufficient evidence was also found for an association between 
Table 5 Results of the best evidence synthesis concerning non-contact and overuse injuries

\begin{tabular}{|c|c|c|c|c|c|}
\hline \multirow[t]{2}{*}{ Risk factor } & \multirow[t]{2}{*}{ Study } & \multirow[t]{2}{*}{ Specification of independent variable } & \multirow{2}{*}{$\begin{array}{l}\text { Outcome } \\
\text { OR/RR (95\% CI) }\end{array}$} & \multicolumn{2}{|c|}{ Best evidence synthesis } \\
\hline & & & & $\begin{array}{l}\text { Presence of } \\
\text { association }\end{array}$ & Level of evidence \\
\hline \multicolumn{6}{|l|}{ Internal load parameters } \\
\hline $\begin{array}{l}\text { Psychosocial } \\
\text { stress and recovery }\end{array}$ & $\begin{array}{l}\text { Brink et al. } \\
\text { [28] }\end{array}$ & $\begin{array}{l}\text { General stress } \\
\text { Emotional stress } \\
\text { Social stress } \\
\text { Conflicts/pressure } \\
\text { Fatigue } \\
\text { Lack of energy } \\
\text { Physical complaints } \\
\text { Success } \\
\text { Social recovery } \\
\text { Physical recovery } \\
\text { General well-being } \\
\text { Sleep quality } \\
\text { Disturbed breaks } \\
\text { Emotional exhaustion } \\
\text { Fitness/injury } \\
\text { Being in shape } \\
\text { Personal accomplishment } \\
\text { Self-efficacy } \\
\text { Self-regulation }\end{array}$ & $\begin{array}{l}1.03(0.75-1.43) \\
1.24(0.85-1.82) \\
1.07(0.74-1.53) \\
0.94(0.70-1.27) \\
0.96(0.70-1.30) \\
1.07(0.75-1.52) \\
1.02(0.71-1.45) \\
0.76(0.55-1.04) \\
0.94(0.71-1.26) \\
0.89(0.66-1.19) \\
0.93(0.68-1.26) \\
0.86(0.64-1.16) \\
1.00(0.73-1.37) \\
0.92(0.69-1.23) \\
1.46(1.09-1.96) \\
0.84(0.64-1.09) \\
0.90(0.68-1.20) \\
0.97(0.74-1.27) \\
0.82(0.66-1.02)\end{array}$ & $\begin{array}{l}\text { No } \\
\text { No } \\
\text { No } \\
\text { No } \\
\text { No } \\
\text { No } \\
\text { No } \\
\text { No } \\
\text { No } \\
\text { No } \\
\text { No } \\
\text { No } \\
\text { No } \\
\text { No } \\
\text { Yes } \\
\text { No } \\
\text { No } \\
\text { No } \\
\text { No }\end{array}$ & Insufficient \\
\hline \multicolumn{6}{|c|}{ External load parameters } \\
\hline \multirow[t]{5}{*}{ Total distance } & \multirow[t]{2}{*}{$\begin{array}{l}\text { Bacon and } \\
\text { Mauger } \\
{[26]}\end{array}$} & 2-week cumulative loads & & & \multirow[t]{5}{*}{$\begin{array}{l}\text { Insufficient } \\
\text { Moderate (2-, 3-, 4- } \\
\text { week cumulative } \\
\text { loads) }\end{array}$} \\
\hline & & $\begin{array}{l}\text { TD_normal load } \\
\text { TD_low load } \\
\text { TD_high load } \\
\text { 3-week cumulative loads } \\
\text { TD_normal load } \\
\text { TD_low load } \\
\text { TD_high load } \\
\text { 4-week cumulative loads } \\
\text { TD—normal load } \\
\text { TD—low load } \\
\text { TD_high load }\end{array}$ & $\begin{array}{l}\text { (ref.) } \\
1.26(0.16-9.77) \\
0.67(0.40-1.14) \\
\text { (ref.) } \\
0.69(0.29-1.64) \\
0.95(0.44-2.05) \\
\text { (ref.) } \\
0.69(0.29-1.64) \\
0.95(0.44-2.05)\end{array}$ & $\begin{array}{l}\text { No } \\
\text { No } \\
\text { No } \\
\text { No } \\
\text { No } \\
\text { No }\end{array}$ & \\
\hline & $\begin{array}{l}\text { Bacon and } \\
\text { Mauger } \\
\text { [26] }\end{array}$ & TD (low, normal, high) & $0.0029(0.0029-0.003)$ & Yes & \\
\hline & \multirow{2}{*}{$\begin{array}{l}\text { Bowen } \\
\text { et al. [27] }\end{array}$} & Accumulated 1/2/3/4-week loads & & & \\
\hline & & $\begin{array}{l}\text { TD_low } \\
\text { TD_-moderate-low } \\
\text { TD_-moderate-high } \\
\text { TD_high } \\
\text { TD_-very high }\end{array}$ & $\begin{array}{l}\mathbf{0 . 3 0} / 0.61 / 0.67 / 1.01 \\
1.45 / 0.95 / 0.87 / 0.77 \\
0.83 / 1.19 / 1.08 / 1.06 \\
1.64 / 1.37 / 1.65 / 1.55 \\
3.04 / 3.35 / 2.79 / 2.30\end{array}$ & $\begin{array}{l}\text { Yes/no/no/no } \\
\text { No/no/no/no } \\
\text { No/no/no/no } \\
\text { No/no/no/no } \\
\text { No/no/no/no }\end{array}$ & \\
\hline \multirow{2}{*}{$\begin{array}{l}\text { High speed } \\
\text { running }\end{array}$} & \multirow{2}{*}{$\begin{array}{l}\text { Bacon and } \\
\text { Mauger } \\
{[26]}\end{array}$} & 2-week cumulative loads & & & \multirow{2}{*}{$\begin{array}{l}\text { Insufficient } \\
\text { Conflicting (2-, 4-week } \\
\text { cumulative HSD loads) } \\
\text { Moderate(3-week HSD } \\
\text { loads) }\end{array}$} \\
\hline & & $\begin{array}{l}\text { HSR_normal load } \\
\text { HSR_low load } \\
\text { HSR_high load } \\
\text { 3-week cumulative loads } \\
\text { HSR_normal load } \\
\text { HSR_low load } \\
\text { HSR_high load } \\
\text { 4-week cumulative loads } \\
\text { HSR_normal load } \\
\text { HSR_-low load } \\
\text { HSR_high load }\end{array}$ & $\begin{array}{l}\text { (ref.) } \\
0.99(0.38-2.59) \\
0.58(0.33-1.02) \\
\text { (ref.) } \\
0.51(0.21-1.21) \\
1.05(0.54-2.03) \\
\text { (ref.) } \\
0.51(0.21-1.21) \\
1.05(0.54-2.03)\end{array}$ & $\begin{array}{l}\text { No } \\
\text { No } \\
\text { No } \\
\text { No } \\
\text { No } \\
\text { No }\end{array}$ & \\
\hline
\end{tabular}


Table 5 Results of the best evidence synthesis concerning non-contact and overuse injuries (Continued)

\begin{tabular}{|c|c|c|c|c|c|}
\hline \multirow[t]{2}{*}{ Risk factor } & \multirow[t]{2}{*}{ Study } & \multirow[t]{2}{*}{ Specification of independent variable } & \multirow{2}{*}{$\begin{array}{l}\text { Outcome } \\
\text { OR/RR }(95 \% \mathrm{Cl})\end{array}$} & \multicolumn{2}{|c|}{ Best evidence synthesis } \\
\hline & & & & $\begin{array}{l}\text { Presence of } \\
\text { association }\end{array}$ & Level of evidence \\
\hline & $\begin{array}{l}\text { Bacon and } \\
\text { Mauger } \\
\text { [26] }\end{array}$ & HSR (low, normal, high) & $0.065(0.064-0.067)$ & Yes & \\
\hline & Bowen & Accumulated 1/2/3/4-week loads & & & \\
\hline & & $\begin{array}{l}\text { HSD_low } \\
\text { HSD_moderately low } \\
\text { HSD_moderately high } \\
\text { HSD_high } \\
\text { HSD_-very high }\end{array}$ & $\begin{array}{l}0.54 / 0.26 / 0.68 / 0.94 \\
1.10 / 0.95 / 0.79 / 0.61 \\
\mathbf{1 . 7 3 / 1 . 4 2 / 1 . 4 0 / 2 . 1 4} \\
0.65 / 1.75 / 1.42 / 0.68 \\
0.00 / 0.00 / 0.00 / 0.59\end{array}$ & $\begin{array}{l}\text { No/yes/no/no } \\
\text { No/no/no/no } \\
\text { Yes/no/no/yes } \\
\text { No/no/no/no } \\
\text { No/no/no/no }\end{array}$ & \\
\hline \multirow[t]{2}{*}{ Acceleration } & \multirow{2}{*}{$\begin{array}{l}\text { Bowen } \\
\text { et al. [27] }\end{array}$} & Accumulated 1/2/3/4-week loads & & & Insufficient \\
\hline & & $\begin{array}{l}\text { ACC-low } \\
\text { ACC - moderately low } \\
\text { ACC-moderately high } \\
\text { ACC - high } \\
\text { ACC - very high }\end{array}$ & $\begin{array}{l}0.47 / 0.60 / 0.69 / 0.95 \\
0.77 / 0.96 / 0.68 / 0.72 \\
1.03 / 1.27 / 1.29 / 1.02 \\
\mathbf{2 . 2 5} / 1.10 / 1.47 / 1.64 \\
1.31 / \mathbf{4 . 2 5} / \mathbf{5 . 1 1 / 4 . 2 5}\end{array}$ & $\begin{array}{l}\text { No/no/no/no } \\
\text { No/no/no/no } \\
\text { No/no/no/no } \\
\text { Yes/no/no/no } \\
\text { No/yes/yes/yes }\end{array}$ & \\
\hline Duration & $\begin{array}{l}\text { Brink et al. } \\
\text { [28] }\end{array}$ & Duration (min) of training sessions and matches & $1.07(0.98-1.18)$ & No & Insufficient \\
\hline \multirow[t]{2}{*}{ Total load } & \multirow{2}{*}{$\begin{array}{l}\text { Bowen } \\
\text { et al. [27] }\end{array}$} & Accumulated 1/2/3/4-week loads & & & Insufficient \\
\hline & & $\begin{array}{l}T L \text {-low } \\
T L \text { - moderately low } \\
T L \text { - moderately high } \\
T L \text { - high } \\
T L \text { - very high }\end{array}$ & $\begin{array}{l}\mathbf{0 . 3 1} / 0.59 / 0.55 / 0.80 \\
1.40 / 1.17 / 0.85 / 1.04 \\
0.79 / 1.13 / 1.37 / 0.94 \\
\mathbf{2 . 2 0 / 1 . 4 5 / 1 . 4 1 / 1 . 6 4} \\
0.00 / 0.00 / 1.39 / 1.07\end{array}$ & $\begin{array}{l}\text { yes/no/no/no } \\
\text { No/no/no/no } \\
\text { No/no/no/no } \\
\text { Yes/no/no/no } \\
\text { No/no/no/no }\end{array}$ & \\
\hline \multirow[t]{2}{*}{ ACWR } & \multirow{2}{*}{$\begin{array}{l}\text { Bowen } \\
\text { et al. [27] }\end{array}$} & Overall/low chronic/high chronic & & & Insufficient \\
\hline & & 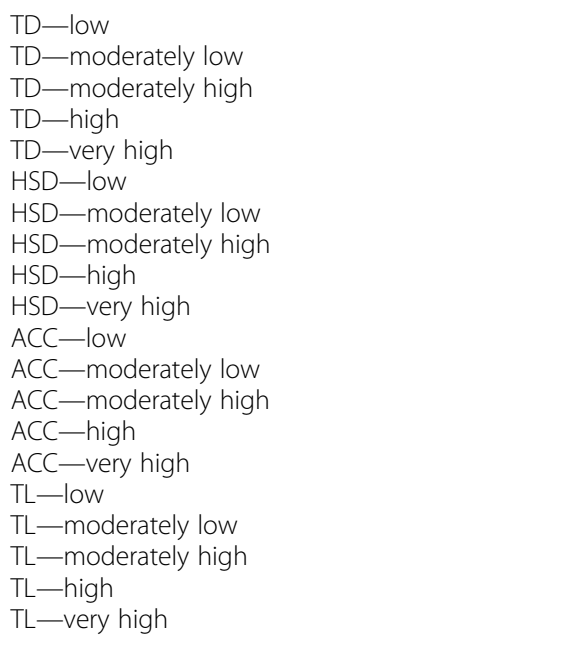 & $\begin{array}{l}1.50 / 0.29 / 1.19 \\
0.96 / 1.12 / 0.62 \\
1.45 / 1.43 / 1.53 \\
1.05 / 1.28 / 1.51 \\
0.00 /-/- \\
0.60 / 0.63 / 1.20 \\
0.88 / 0.88 / 0.81 \\
1.33 / 0.85 / 2.09 \\
1.39 / 2.55 / 0.47 \\
-/-/- \\
1.22 / 0.31 / 1.37 \\
0.81 / 1.32 / 0.63 \\
1.52 / 1.23 / 1.49 \\
1.41 / 1.30 / 1.54 \\
-/-/- \\
1.20 / 0.50 / 0.98 \\
0.84 / 0.84 / 0.79 \\
\mathbf{1 . 8 7} / 1.55 / 1.93 \\
0.87 / 1.16 / 0.53 \\
-/-/-\end{array}$ & $\begin{array}{l}\text { No/no/no } \\
\text { No/no/no } \\
\text { No/no/no } \\
\text { No/no/no } \\
\text { No/n.a./n.a. } \\
\text { No/no/no } \\
\text { No/no/no } \\
\text { No/no/yes } \\
\text { No/yes/no } \\
\text { n.a./n.a./n.a } \\
\text { No/no/no } \\
\text { No/no/no } \\
\text { No/no/no } \\
\text { No/no/no } \\
\text { n.a./n.a./n.a } \\
\text { No/no/no } \\
\text { No/no/no } \\
\text { Yes/no/no } \\
\text { No/no/no } \\
\text { n.a./n.a./n.a }\end{array}$ & \\
\hline \multicolumn{6}{|c|}{ Combined internal/external load parameters } \\
\hline Workload & $\begin{array}{l}\text { Brink et al. } \\
\text { [28] }\end{array}$ & Product of the session-RPE and duration & $1.01(1.00-1.02)$ & No & Insufficient \\
\hline Monotony & $\begin{array}{l}\text { Brink et al. } \\
{[28]}\end{array}$ & $\begin{array}{l}\text { Daily mean training load divided by the SD of } \\
\text { the daily mean training load over a 1-week } \\
\text { period }\end{array}$ & $0.84(0.25-2.76)$ & No & Insufficient \\
\hline Strain & $\begin{array}{l}\text { Brink et al. } \\
\text { [28] }\end{array}$ & Product of weekly training load and monotony & $1.00(1.00-1.01)$ & No & Insufficient \\
\hline
\end{tabular}

In bold are statistically significant $(p<0.05)$ associations

$O R$ odds ratio, $R R$ risk ratio, $C l$ confidence interval, ref reference, TD total distance, HSD high speed distance, $A C C$ acceleration, $T L$ total load, $H S R$ high speed running, min minutes, ACWR Acute Chronic Workload Ratio, n.a. not applicable, RPE rate of perceived exertion, $S D$ standard deviation 
Table 6 Results of the best evidence synthesis concerning all injuries

\begin{tabular}{|c|c|c|c|c|c|}
\hline \multirow[t]{2}{*}{ Risk factor } & \multirow[t]{2}{*}{ Study } & \multirow[t]{2}{*}{ Specification of independent variable } & \multirow{2}{*}{$\begin{array}{l}\text { Outcome } \\
\text { OR/RR (95\% Cl) }\end{array}$} & \multicolumn{2}{|c|}{ Best evidence synthesis } \\
\hline & & & & $\begin{array}{l}\text { Presence of } \\
\text { association }\end{array}$ & $\begin{array}{l}\text { Level of } \\
\text { evidence }\end{array}$ \\
\hline \multicolumn{6}{|c|}{ External load parameters } \\
\hline \multirow{2}{*}{$\begin{array}{l}\text { Total } \\
\text { distance }\end{array}$} & \multirow{2}{*}{$\begin{array}{l}\text { Bowen } \\
\text { et al. [27] }\end{array}$} & Accumulated 1/2/3/4-week loads & & & \multirow[t]{2}{*}{ Insufficient } \\
\hline & & $\begin{array}{l}\text { TD_low } \\
\text { TD_moderately low } \\
\text { TD_-moderately high } \\
\text { TD_high } \\
\text { TD_very high }\end{array}$ & $\begin{array}{l}\mathbf{0 . 2 5} / 0.62 / 0.53 / 0.89 \\
1.38 / 0.76 / 1.23 / 0.73 \\
0.95 / \mathbf{1 . 5 5} / 1.36 / 1.19 \\
1.57 / 1.27 / 1.31 / \mathbf{1 . 6 4} \\
2.59 / 2.88 / 2.37 / 1.29\end{array}$ & $\begin{array}{l}\text { Yes/no/no/no } \\
\text { No/no/no/no } \\
\text { No/yes/no/no } \\
\text { No/no/no/yes } \\
\text { No/no/no/no }\end{array}$ & \\
\hline \multirow{2}{*}{$\begin{array}{l}\text { High speed } \\
\text { running }\end{array}$} & \multirow{2}{*}{$\begin{array}{l}\text { Bowen } \\
\text { et al. [27] }\end{array}$} & Accumulated 1/2/3/4-week loads & & & \multirow{2}{*}{ Insufficient } \\
\hline & & $\begin{array}{l}\text { HSD_low } \\
\text { HSD - moderately low } \\
\text { HSD_-moderately high } \\
\text { HSD_high } \\
\text { HSD_-very high }\end{array}$ & $\begin{array}{l}\mathbf{0 . 3 8} / \mathbf{0 . 3 0} / 0.67 / 0.79 \\
1.16 / 0.81 / 0.84 / 0.73 \\
\mathbf{1 . 7 3 / 1 . 7 2} / 1.15 / \mathbf{1 . 5 6} \\
0.59 / 1.45 / \mathbf{1 . 6 6 / 1 . 2 6} \\
0.82 / 0.00 / 0.33 / 0.33\end{array}$ & $\begin{array}{l}\text { Yes/yes/no/no } \\
\text { No/no/no/no } \\
\text { Yes/yes/no/yes } \\
\text { No/no/yes/no } \\
\text { No/no/no/no }\end{array}$ & \\
\hline \multirow{2}{*}{ Acceleration } & \multirow{2}{*}{$\begin{array}{l}\text { Bowen } \\
\text { et al. [27] }\end{array}$} & Accumulated 1/2/3/4-week loads & & & \multirow[t]{2}{*}{ Insufficient } \\
\hline & & $\begin{array}{l}\text { ACC-low } \\
\text { ACC - moderately low } \\
\text { ACC-moderately high } \\
\text { ACC-high } \\
\text { ACC - very high }\end{array}$ & $\begin{array}{l}\mathbf{0 . 3 5} / 0.51 / 0.63 / 0.93 \\
1.01 / 0.92 / 0.77 / 0.82 \\
1.00 / 1.21 / 1.32 / 1.01 \\
\mathbf{1 . 8 3 / 1 . 3 7 / 1 . 3 8 / 1 . 6 6} \\
\mathbf{3 . 0 6 / 3 . 1 9 / 3 . 8 4 / 2 . 3 7}\end{array}$ & $\begin{array}{l}\text { Yes/no/no/no } \\
\text { No/no/no/no } \\
\text { No/no/no/no } \\
\text { Yes/no/no/yes } \\
\text { Yes/yes/yes/no }\end{array}$ & \\
\hline \multirow[t]{2}{*}{ Total load } & \multirow{2}{*}{$\begin{array}{l}\text { Bowen } \\
\text { et al. [27] }\end{array}$} & Accumulated 1/2/3/4-week loads & & & \multirow{2}{*}{ Insufficient } \\
\hline & & $\begin{array}{l}T L \text {-low } \\
T L \text { - moderately low } \\
T L \text { - moderately high } \\
T L \text { - high } \\
T L \text { - very high }\end{array}$ & $\begin{array}{l}\mathbf{0 . 2 7} / 0.50 / 0.55 / 0.75 \\
1.45 / 1.07 / 0.98 / 1.01 \\
0.98 / 1.38 / 1.39 / 1.12 \\
\mathbf{1 . 6 5 / 1 . 0 3 / 1 . 0 9 / 1 . 2 0} \\
\text { 2.00/1.93/1.59/1.84 }\end{array}$ & $\begin{array}{l}\text { Yes/no/no/no } \\
\text { No/no/no/no } \\
\text { No/no/no/no } \\
\text { Yes/no/no/no } \\
\text { No/no/no/no }\end{array}$ & \\
\hline \multirow[t]{2}{*}{ ACWR } & \multirow{2}{*}{$\begin{array}{l}\text { Bowen } \\
\text { et al. [27] }\end{array}$} & Overall/low chronic/high chronic & & & \multirow[t]{2}{*}{ Insufficient } \\
\hline & & 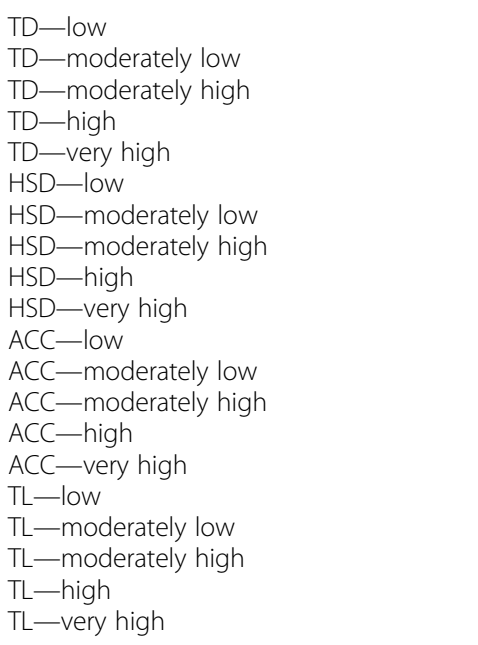 & $\begin{array}{l}1.00 / \mathbf{0 . 2 8} / 0.91 \\
1.25 / 1.43 / 0.98 \\
0.97 / 0.97 / 1.19 \\
1.13 / 1.76 / 1.21 \\
2.09 /-/ 1.80 \\
\mathbf{0 . 4 7} / 0.47 / 1.52 \\
1.10 / 0.86 / 1.11 \\
1.32 / 1.30 / 1.27 \\
0.98 / 1.82 / 0.50 \\
0.95 /-/ 1.63 \\
0.85 / 0.29 / 0.71 \\
1.16 / 1.49 / 1.04 \\
1.15 / 0.94 / 1.25 \\
1.44 / 1.70 / 1.10 \\
2.09 /-/ 2.71 \\
0.84 / 0.37 / 0.81 \\
1.15 / 1.15 / 1.22 \\
1.34 / 1.16 / 1.34 \\
1.01 / 1.59 / 0.43 \\
1.17 /-/ 2.67\end{array}$ & $\begin{array}{l}\text { No/yes/no } \\
\text { No/no/no } \\
\text { No/no/no } \\
\text { No/no/no } \\
\text { No/n.a./no } \\
\text { Yes/no/no } \\
\text { No/no/no } \\
\text { No/no/no } \\
\text { No/no/no } \\
\text { No/n.a./no } \\
\text { No/yes/no } \\
\text { No/no/no } \\
\text { No/no/no } \\
\text { No/no/no } \\
\text { No/n.a./no } \\
\text { No/no/no } \\
\text { No/no/no } \\
\text { No/no/no } \\
\text { No/no/no } \\
\text { No/n.a./no }\end{array}$ & \\
\hline \multicolumn{6}{|c|}{ Combined internal/external load parameters } \\
\hline \multirow[t]{2}{*}{ Workload } & \multirow{2}{*}{$\begin{array}{l}\text { Delecroix } \\
\text { et al. [29] }\end{array}$} & U19 & & & \multirow{2}{*}{$\begin{array}{l}\text { Insufficient } \\
\text { Moderate (1- } \\
\text { week workload) }\end{array}$} \\
\hline & & $\begin{array}{l}\text { 1-week workload } \\
\text { 2-week cumulative workload } \\
\text { 3-week cumulative workload } \\
\text { 4-week cumulative workload } \\
\text { U21 } \\
\text { 1-week workload } \\
\text { 2-week cumulative workload } \\
\text { 3-week cumulative workload }\end{array}$ & 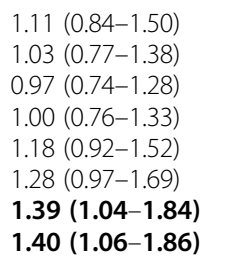 & $\begin{array}{l}\text { No } \\
\text { No } \\
\text { No } \\
\text { No } \\
\text { No } \\
\text { No } \\
\text { Yes } \\
\text { Yes }\end{array}$ & \\
\hline
\end{tabular}


Table $\mathbf{6}$ Results of the best evidence synthesis concerning all injuries (Continued)

\begin{tabular}{|c|c|c|c|c|c|}
\hline \multirow[t]{2}{*}{ Risk factor } & \multirow[t]{2}{*}{ Study } & \multirow[t]{2}{*}{ Specification of independent variable } & \multirow{2}{*}{$\begin{array}{l}\text { Outcome } \\
\text { OR/RR }(95 \% \mathrm{Cl})\end{array}$} & \multicolumn{2}{|c|}{ Best evidence synthesis } \\
\hline & & & & $\begin{array}{l}\text { Presence of } \\
\text { association }\end{array}$ & $\begin{array}{l}\text { Level of } \\
\text { evidence }\end{array}$ \\
\hline & $\begin{array}{l}\text { Raya- } \\
\text { Gonzálaz } \\
\text { et al. [30] }\end{array}$ & $\begin{array}{l}\text { Sum of load (RPE* duration) for all training sessions and matches } \\
\text { for each week }\end{array}$ & $1.00(0.99-1.00)^{\mathrm{a}}$ & No & \\
\hline \multirow[t]{3}{*}{ ACWR } & \multirow{2}{*}{$\begin{array}{l}\text { Delecroix } \\
\text { et al. [29] }\end{array}$} & U19 & & & \multirow{2}{*}{$\begin{array}{l}\text { Insufficient } \\
\text { Moderate }(4- \\
\text { week A:C } \\
\text { workload) }\end{array}$} \\
\hline & & $\begin{array}{l}\text { 2-week A:C workload } \\
\text { 3-week A:C workload } \\
\text { 4-week A:C workload } \\
\text { Week-to-week workload changes } \\
\text { U21 } \\
\text { 2-week A:C workload } \\
\text { 3-week A:C workload } \\
\text { 4-week A:C workload } \\
\text { Week-to-week workload changes }\end{array}$ & $\begin{array}{l}0.99(0.90-1.09) \\
1.01(0.95-1.06) \\
1.01(0.96-1.07) \\
1.00(0.96-1.04) \\
0.86(0.58-1.29) \\
0.88(0.66-1.16) \\
0.89(0.71-1.13) \\
1.00(0.95-1.06)\end{array}$ & $\begin{array}{l}\text { No } \\
\text { No } \\
\text { No } \\
\text { No } \\
\text { No } \\
\text { No } \\
\text { No } \\
\text { No }\end{array}$ & \\
\hline & $\begin{array}{l}\text { Raya- } \\
\text { Gonzálaz } \\
\text { et al. [30] }\end{array}$ & $\begin{array}{l}\text { Sum of the current week's load (acute load) divided by the } \\
\text { average weekly training load over the previous four weeks } \\
\text { (chronic load) }\end{array}$ & $0.16(0.01-1.84)^{a}$ & No & \\
\hline
\end{tabular}

In bold are statistically significant $(p<0.05)$ associations

$O R$ odds ratio, $R R$ risk ratio, $C l$ confidence interval, ref reference, TD total distance, HSD high speed distance, $A C C$ acceleration, $T L$ total load, $H S R$ high speed running, min minutes, $A C W R$ acute to chronic workload ratio, n.a. not applicable, RPE rate of perceived exertion, SD standard deviation, U19 under 19 years squad, $U 21$ under 21 years squad, $A: C$ acute to chronic

${ }^{\mathrm{a}} 90 \%$ confidence interval

very high ACC (> 9254), accumulated over $2-4$ weeks, and high weekly ACC (2558-3474) loads and the occurrence of non-contact/overuse injuries. Moreover, there is insufficient evidence for an association between (1) low weekly ACC loads, (2) high 1- and 4-weekly ACC loads, and (3) very high 1-3-weekly ACC loads and overall injury occurrence. For all other comparisons, insufficient evidence exists for a lack of an association.

\section{Duration}

One high-quality study evaluated the association between the duration of training and matches (over the preceding week, calculated in hours) and traumatic and overuse injury risk [28]. Weekly duration was significantly higher for players with a traumatic injury compared to healthy players, resulting in insufficient evidence for an association as only one study reported this. Insufficient evidence also exists for no correlation between duration and overuse injury risk.

\section{Acute to Chronic Workload Ratio (ACWR)}

One high-quality study investigated the association between A:C workload ratios (based on GPS and accelerometer-derived variables) and the occurrence of contact/traumatic, non-contact/overuse, and overall injuries [27]. The risk of contact injury was increased when the overall A:C total distance (TD) ratio was $\geq 1.76$. For low chronic TDs covered (<22 $335 \mathrm{~m}$ ), overall injury occurrence was associated with a low A:C TD ratio (0-0.32). In addition, there is insufficient evidence for (1) an association between very high A:C TD ratios and contact injury occurrence, (2) an association between a low A:C TD ratio and overall injury occurrence (low chronic TD covered), and (3) no association for all other comparisons.

For low chronic high speed running distance (HSD) $(<$ $938 \mathrm{~m})$, non-contact injury risk was increased by a high $\mathrm{A}: \mathrm{C}$ ratio (1.41-1.96). For high chronic HSDs (> $938 \mathrm{~m}$ ), non-contact injury occurrence was associated with a moderately high A:C ratio (0.91-1.34). However, a low ratio (0-0.36) for all chronic HSDs was associated with a reduced overall injury risk. Moreover, there is insufficient evidence for (1) an association between high A:C HSD ratios and non-contact injury occurrence (low chronic HSDs), (2) an association between a moderately high A:C HSD ratio and non-contact injury occurrence (high chronic HSDs), (3) an association between low A: $\mathrm{C}$ HSD ratios and overall injury occurrence, and (4) no association for all other comparisons.

The risk of contact injury was increased when the A:C acceleration (ACC) ratio was $>1.77$ (very high). Overall injury risk was reduced when the $\mathrm{A}: \mathrm{C} \mathrm{ACC}$ ratio was low (0-0.33) for low chronic ACC (< 1856). Furthermore, there is insufficient evidence for (1) an association between very high $\mathrm{A}: \mathrm{C} \mathrm{ACC}$ ratios and contact injury occurrence, (2) an association between a low A:C ACC ratio and reduced overall injury occurrence (low chronic ACCs), and (3) no association for all other comparisons.

The risk of non-contact injury was increased when the A:C total load (TL) ratio was moderately high $(0.88$ 1.32). A moderately low A:C TL ratio (0.44-0.88) was associated with the occurrence of contact injuries. In sum, there is insufficient evidence for (1) an association 
between moderately high A:C TL ratios and non-contact injury occurrence, (2) an association between moderately low A:C TL ratios and contact injury occurrence, and (3) no association for all other comparisons.

\section{Evaluation of Combined Internal and External Load Parameters \\ Workload}

Three studies, one high quality and two low quality, investigated workload as a risk factor for injury occurrence [28-30]. All three studies defined workload as the product of the rate of perceived exertion (sRPE) during a session and the session's duration. In the studies of Delecroix et al. and Raya-González et al., the sRPE was measured using a 10-point scale $[29,30]$, while Brink and colleagues used a 15-point Borg scale [28]. There was insufficient evidence for an association between workload and contact/traumatic injuries and also insufficient evidence for no association between workload and non-contact/overuse injuries [28].

There was moderate evidence for a lack of an association between 1-week workloads and overall injury risk $[29,30]$. Delecroix and colleagues also calculated 2-, 3-, and 4-week cumulative workloads and found an association for 3- and 4-week cumulative workloads and injury occurrence in their under-21 (U21) squad, resulting in insufficient evidence for an association between 3- and 4-week cumulative workloads, and insufficient evidence for no association between 2- week cumulative workloads and overall injuries [29].

\section{Monotony}

One high-quality study described the association between the monotony of training load and the occurrence of traumatic injuries, as well as overuse injuries [28]. There is insufficient evidence for an association between monotony and traumatic injuries, and insufficient evidence for no association between monotony and overuse injuries.

\section{Strain}

There is insufficient evidence for an association between the amount of effort (strain) a player experienced and the occurrence of traumatic injuries, and also insufficient evidence for no association between strain and overuse injuries [28].

\section{Acute to Chronic Workload Ratio (ACWR)}

Two low-quality studies investigated the association between the acute to chronic $(\mathrm{A}: \mathrm{C})$ workload ratio and the occurrence of overall injuries [29, 30]. Delecroix et al. calculated A:C ratios (week-to-week, 2 weeks, 3 weeks, and 4 weeks) by dividing the 1-week workload (product of the SRPE and duration of training session and matches) by the average workload of the last 28/21/14 days [29]. In the under-19s (U19), as well as in the U21 squad, no associations were found. Raya-González and colleagues found no association between A:C workload $(\mathrm{sRPE} \times$ duration) ratios (where acute refers to the current week and chronic refers to the rolling 4-week average) and injury occurrence [30]. This resulted in moderate evidence for no association between A:C workload ratios (4 weeks) and the occurrence of injuries and insufficient evidence for no association for $\mathrm{A}: \mathrm{C}$ ratios (week-to-week, 2 weeks, and 3 weeks).

\section{Discussion}

This systematic review evaluates the evidence concerning the effects of internal and external training load on injury risk in elite youth soccer players. According to the best evidence synthesis, there was insufficient evidence for an association between psychosocial stress and recovery (internal load) and the occurrence of injuries.

With regard to external load parameters, there was moderate evidence for no association between 2-, 3-, and 4-week cumulative loads for total distance covered and injury risk. Conflicting evidence exists for the association between 2- and 4-week accumulated HSD loads and non-contact/overuse injury risk. Moreover, there was moderate evidence for a lack of an association between 3-weekly accumulated HSD loads and injury occurrence. Furthermore, insufficient evidence existed for an association between the number of accelerations, duration of training/matches, $\mathrm{A}: \mathrm{C}$ workload ratios (based on GPS and accelerometer-derived variables), and the occurrence of injuries.

Combined load parameters, such as monotony and strain, showed insufficient evidence for an association with injury risk. There was moderate evidence for no correlation between 1-week workloads (product of sRPE and the duration) and overall injury risk, alongside insufficient evidence for an association between 3- and 4-week cumulative workloads and injuries. Finally, moderate evidence existed for a lack of an association between A:C workload ratios (4 weeks) and the occurrence of injuries, with insufficient evidence also found for no association for A:C ratios (week-to-week, 2 weeks, and 3 weeks).

Overall, the evidence for associations between internal/external load factors and the occurrence of injuries is based on a limited number of studies $(n=5)$, with a maximum of three studies per potential risk factor, which limits the conclusions that can be drawn.

In addition, the risk of bias assessment resulted in two studies being assessed as having a low risk of bias and three studies being assessed as having a high risk of bias. Critical items in the risk of bias assessment were the items on absence of injuries at the start of the study (item 4) and adequacy of follow-up of cohorts (item 7). 
None of the studies demonstrated that included soccer players were injury-free at baseline. Moreover, none of the studies reported a follow-up rate or provided a description of those soccer players who were lost between inception and follow-up. These studies are therefore more susceptible to selection and attrition bias, and, as a consequence, this will affect the generalizability of our results.

In addition to the shortcomings mentioned above, there are some other explanations for the low evidence found in this systematic review. All included studies used a general injury definition, for example "Any physical complaint sustained by a player that results from a soccer match or training, resulting in time loss or medical attention" or "A non-contact/contact injury was defined as one that caused any absence from future soccer participation" [27, 28]. Using a general injury definition will lead to the inclusion of all types of injuries. For example, muscle injuries are the most common noncontact related (overuse) injuries in soccer and constitute approximately 30 to $60 \%$ of all time-loss injuries in elite youth soccer players [14, 31-33]. Selecting a more homogenous group of injured players based on a more specific injury definition (i.e., muscle injuries), taking into account the injury mechanism, will probably lead to a clearer view on the true association between workload and injury risk. Furthermore, there is a possibility that the association between training load and injury risk alone is not that strong and that other factors, the interplay between them, or the mediating effects of them are more important in a population of elite youth soccer players. This is underlined by Pfirrmann and colleagues who conducted a systematic review to compare the injury incidences and characteristics of male professional adult and elite youth soccer players [34]. They summarized that factors, such as age, playing position, season, concealment of injury, multiple injuries, imbalance between external pressure and internal effort, training, recovery time, re-injuries, and maturity status, can lead to higher injury incidences. The multifactorial nature of injuries needs to be addressed, which is confirmed by the model of Meeuwisse et al. that highlighted the importance of accounting for all factors involved, such as the internal and external risk factors as well as the inciting event $[35,36]$. Future studies investigating the association between internal and external training load and injury risk should also take these factors into account, and use a multifactorial approach to unravel the association between several risk factors and injuries.

To our knowledge, this is the first systematic review summarizing the available evidence for associations between internal and external training load and injury occurrence in elite youth soccer players. A strength of this systematic review is that a comprehensive literature search was conducted in five different databases, which makes it likely that all potential relevant studies have been identified. Alongside this, a best evidence synthesis was applied to summarize and evaluate the existing evidence. This methodology results in transparency in the process of evidence assessment by applying clear criteria that include the quality of the studies. However, this study also has some limitations. First, the studies included in this systematic review only included elite youth soccer players from Europe (UK, Spain, France, and the Netherlands), resulting in more homogenous groups of soccer players and possibly reducing generalizability to other parts of the world. Therefore, we would like to emphasize that more research is needed regarding internal and external workload and injury risk in elite youth soccer players. Second, different operational definitions of injury are used in the included studies. This variability of definitions can impact injury estimates and, as a result, influence the association between training load and injury risk. Third, there is the potential for a publication and language bias given that only published literature, written in English, was included for the purposes of this review.

\section{Conclusion}

After summarizing the literature, it can be concluded that there is a paucity of evidence for an association between internal and external training load parameters and injury risk in elite youth soccer players. Future highquality longitudinal research is required to further assess the role of internal and external training load on the occurrence of injuries in elite youth soccer players, where a multifactorial approach must also be applied. In addition, the shortcomings of the included studies concerning selection and attrition bias should be taken into account.

\section{Supplementary Information}

The online version contains supplementary material available at https://doi. org/10.1186/s40798-020-00296-1.

Additional file 1. Search strategy

\section{Acknowledgements}

The authors would like to thank Wichor Bramer of the Erasmus MC Medical Library for developing and updating the search strategies.

\section{Authors' Contributions}

All 4 authors made a substantial contribution to the information and material submitted and have read and approved the final version. SV performed the literature searches, selected the articles for inclusion, extracted relevant information from the included studies, and wrote the manuscript. RMvR selected the articles for inclusion, extracted relevant information from the included studies, performed data analysis, and wrote the manuscript. RC assisted with writing the manuscript. JHS performed the data analysis and contributed to the content of the article. 


\section{Funding}

This work was supported by the National Association of Applied Sciences SIA (grant numbers: RAAK.PRO02.022 and SPR.VG01.007).

\section{Availability of Data and Materials}

Not applicable.

\section{Ethics Approval and Consent to Participate}

Not applicable.

\section{Consent for Publication}

Not applicable.

\section{Competing Interests}

The authors, Sven Verstappen, Rogier M van Rijn, Rick Cost, and Janine H

Stubbe, declare that they have no competing interests.

\section{Author details}

${ }^{1}$ Codarts, University of the Arts, Kruisplein 26, 3012 CC Rotterdam, The Netherlands. ${ }^{2}$ PErforming artist and Athlete Research Lab (PEARL), Rotterdam, The Netherlands. ${ }^{3}$ Feyenoord Academy, Rotterdam, The Netherlands. ${ }^{4}$ Feyenoord, Rotterdam, The Netherlands. ${ }^{5}$ Erasmus MC University Medical Center, Department of General Practice, Rotterdam, The Netherlands.

${ }^{6}$ Rotterdam Arts and Science Lab (RASL), Rotterdam, The Netherlands.

\section{Received: 14 August 2020 Accepted: 20 December 2020}

\section{Published online: 11 January 2021}

\section{References}

1. Barnes C, Archer DT, Hogg B, Bush M, Bradley PS. The evolution of physical and technical performance parameters in the English Premier League. Int Sports Med. 2014 Dec;35(13):1095-100.

2. Kellmann $M$, Bertollo M, Bosquet $L$, Brink $M$, Coutts AJ, Duffield R, et al. Recovery and performance in sport: consensus statement. Int J Sports Physiol Perform. 2018 Feb 1;13(2):240-5.

3. Kentta G, Hassmen P. Overtraining and recovery. A conceptual model. Sports Med. 1998 Jul;26(1):1-16.

4. Ekstrand J, Hagglund M, Walden M. Epidemiology of muscle injuries in professional football (soccer). Am J Sports Med. 2011 Jun;39(6):1226-32.

5. Walden M, Hagglund M, Ekstrand J. UEFA Champions League study: a prospective study of injuries in professional football during the 2001-2002 season. Br J Sports Med. 2005 Aug;39(8):542-6.

6. Gabbett TJ, Whyte DG, Hartwig TB, Wescombe H, Naughton GA. The relationship between workloads, physical performance, injury and illness in adolescent male football players. Sports Med. 2014 Jul;44(7):989-1003.

7. Roksund OD, Kristoffersen M, Bogen BE, Wisnes A, Engeseth MS, Nilsen AK, et al. Higher drop in speed during a repeated sprint test in soccer players reporting former hamstring strain injury. Front Physiol. 2017:8:25.

8. Rowland TW. Effect of prolonged inactivity on aerobic fitness of children. J Sports Med Phys Fitness. 1994;34(2):147-55.

9. Drawer S, Fuller CW. Propensity for osteoarthritis and lower limb joint pain in retired professional soccer players. Br J Sports Med. 2001 Dec;35(6):402-8.

10. Fyfe JJ, Opar DA, Williams MD, Shield AJ. The role of neuromuscular inhibition in hamstring strain injury recurrence. J Electromyogr Kinesiol. 2013 Jun:23(3):523-30

11. Leventer L, Eek F, Hofstetter S, Lames M. Injury patterns among elite football players: a media-based analysis over 6 seasons with emphasis on playing position. Int J Sports Med. 2016 Oct;37(11):898-908.

12. Maffulli N, Longo UG, Gougoulias N, Loppini $M$, Denaro V. Long-term health outcomes of youth sports injuries. Br J Sports Med. 2010;44(1):21-5.

13. Junge A, Chomiak J, Dvorak J. Incidence of football injuries in youth players. Comparison of players from two European regions. Am J Sports Med. 2000; 28(5 Suppl):S47-50.

14. Ergun M, Denerel HN, Binnet MS, Ertat KA. Injuries in elite youth football players: a prospective three-year study. Acta Orthop Traumatol Turc. 2013, 47(5):339-46.

15. Impellizzeri FM, Marcora SM, Coutts AJ. Internal and external training load: 15 years on. Int J Sports Physiol Perform. 2019 Feb 1;14(2):270-3.

16. Ehrmann FE, Duncan CS, Sindhusake D, Franzsen WN, Greene DA. GPS and injury prevention in professional soccer. J Strength Cond Res. 2016 Feb; 30(2):360-7.
17. Jaspers A, Kuyvenhoven JP, Staes F, Frencken WGP, Helsen WF, Brink MS Examination of the external and internal load indicators' association with overuse injuries in professional soccer players. J Sci Med Sport. 2018 Jun; 21(6):579-85.

18. Malone S, Owen A, Newton M, Mendes B, Collins KD, Gabbett TJ. The acute: chonic workload ratio in relation to injury risk in professional soccer. J Sci Med Sport. 2017 Jun;20(6):561-5.

19. McCall A, Dupont G, Ekstrand J. Internal workload and non-contact injury: a one-season study of five teams from the UEFA Elite Club Injury Study. Br J Sports Med. 2018 Dec;52(23):1517-22

20. Owen AL, Forsyth JJ, del Wong P, Dellal A, Connelly SP, Chamari K. Heart rate-based training intensity and its impact on injury incidence among elitelevel professional soccer players. J Strength Cond Res. 2015;29(6):1705-12.

21. Moher D, Liberati A, Tetzlaff J, Altman DG, Group P. Preferred reporting items for systematic reviews and meta-analyses: the PRISMA statement. PLoS Med. 2009;6(7):e1000097.

22. Soligard T, Schwellnus M, Alonso JM, Bahr R, Clarsen B, Dijkstra HP, et al. How much is too much? (Part 1) International Olympic Committee consensus statement on load in sport and risk of injury. Br J Sports Med. 2016 Sep:50(17):1030-41.

23. Wells GA, Shea B, O'Connell D, Peterson J, Welch V, Losos M, et al. The Newcastle-Ottawa Scale (NOS) for assessing the quality of nonrandomised studies in meta-analyses. retrieved from: http://www.ohrica/programs/ clinical epidemiology/oxfordasp. Retrieved on the 15th of June 2020.

24. Lopez-Valenciano A, Ruiz-Perez I, Garcia-Gomez A, Vera-Garcia FJ, De Ste CM, Myer GD, et al. Epidemiology of injuries in professional football: a systematic review and meta-analysis. Br J Sports Med. 2020;54(12):711-8.

25. van der Heide I, van Rijn RM, Robroek SJ, Burdorf A, Proper KI. Is retirement good for your health? A systematic review of longitudinal studies. BMC Public Health. 2013:13:1180.

26. Bacon CS, Mauger AR. Prediction of overuse injuries in professional U18-U21 footballers using metrics of training distance and intensity. J Strength Cond Res. 2017:31(11):3067-76.

27. Bowen L, Gross AS, Gimpel M, Li FX. Accumulated workloads and the acute: chronic workload ratio relate to injury risk in elite youth football players. $\mathrm{Br}$ Sports Med. 2017;51(5):452-9.

28. Brink MS, Visscher C, Arends S, Zwerver J, Post WJ, Lemmink KA. Monitoring stress and recovery: new insights for the prevention of injuries and illnesses in elite youth soccer players. Br J Sports Med. 2010;44(11):809-15.

29. Delecroix B, Delaval B, Dawson B, Berthoin S, Dupont G. Workload and injury incidence in elite football academy players. J Sports Sci. 2019 Dec 37(24):2768-73

30. Raya-González J, Nakamura FY, Castillo D, Yanci J, Fanchini M. Determining the relationship between internal load markers and noncontact injuries in young elite soccer players. Int J Sports Physiol Perform. 2019;14(4):421-5.

31. Brito J, Malina RM, Seabra A, Massada JL, Soares JM, Krustrup P, et al. Injuries in Portuguese youth soccer players during training and match play. J Athl Train. 2012 Mar-Apr:47(2):191-7.

32. Cezarino LG, Gruninger B, Scattone SR. Injury profile in a Brazilian firstdivision youth soccer team: a prospective study. J Athl Train. 2020;55(3): 295-302.

33. Renshaw A, Goodwin PC. Injury incidence in a Premier League youth soccer academy using the consensus statement: a prospective cohort study. BMJ Open Sport Exerc Med. 2016;2(1):e000132

34. Pfirrmann $D$, Herbst $M$, Ingelfinger $P$, Simon $P$, Tug $S$. Analysis of injury incidences in male professional adult and elite yuth soccer players: a systematic review. J Athl Train. 2016 May;51(5):410-24.

35. Bahr R, Krosshaug T. Understanding injury mechanisms: a key component of preventing injuries in sport. Br J Sports Med. 2005;39(6):324-9.

36. Meeuwisse WH, Tyreman H, Hagel B, Emery C. A dynamic model of etiology in sport injury: the recursive nature of risk and causation. Clin J Sport Med. 2007 May; 17(3):215-9.

\section{Publisher's Note}

Springer Nature remains neutral with regard to jurisdictional claims in published maps and institutional affiliations. 